\begin{tabular}{|c|c|c} 
Eiszeitalter u. Gegenwart & $\mathbf{3 3}$ & $\begin{array}{c}119-132 \\
\text { 6 Abb., 2 Tab. }\end{array}$
\end{tabular}

\title{
Sedimentologische Zuordnung saalezeitlicher Gletscherablagerungen zu mehreren Vorstößen am unteren Niederrhein
}

\author{
Helmut Siebertz *
}
Classification, glacifluvial deposit, glaciation, morain, Saale Ice Age, alluvial fan, sand, grain size, nomogram, pit sand, borehole section.
Lower Rhine Westphalian Basin, Kalkar Area, North Rhine Westfalia. TK 25: Nr. 4203

Kurzfassung: Beim Vorstoß sowie Rückzug des Eises werden Sandersedimente abgelagert. Sie setzen sich aus gröberen Vorschütt- und feineren Nachschüttsanden zusammen, die (meistens) durch eine Grundmoräne voneinander getrennt sind. Vorschüttsand, Grundmoräne und Nachschüttsand bilden einen glaziären Zyklus. Fehlt die Grundmoräne, so ist eine genetische Gliederung der Sedimente in eine Vorstoß- und Rückzugsphase erschwert.

Bei der Sedimentuntersuchung zeigte sich, daß einzelne Korngrößenfraktionen für eine Gliederung des Sanders kaum von Bedeutung sind. Die Zusammenfassung von Korngrößenfraktionen zu Korngrößengruppen (Tab. 1) ermöglichte eine exakte Gliederung der Sandersedimente und damit die genetische Ansprache der Gletscherablagerungen am unteren Niederrhein.

Aufgrund der Einlagerung von Grundmoränenmaterial in den Sandersedimenten konnten für das Drenthe-Stadial im Raum Louisendorf-Moyland drei Eisvorstöße nachgewiesen werden.

\section{[Sedimentological Classification of Saale-Ice-Age Glacier Deposits on the Lower Rhine into Several Periods of Ice Advance]}

Abstract: Glacio-fluvial sands are deposited when ice advances or recedes, which consist of coarse sands from the melting of the advancing glacier (Vorschüttsand) and finer sands deposited during the ice recession (Nachschüttsand); these are usually seperated by a ground moraine. Sand sediments from the ice advance, a ground moraine, and sand from the ice recession form a glacial cycle. If there is no ground moraine, a genetical classification of the sediments into the phases of advance and recession is more difficult.

When the sediments were examined, it was found that certain grain-size categories were hardly significant for a classification of the sands. By forming larger groups out of the individual grain-size categories (Fig. 1) a precise classification of the glacio-fluvial sediments was made possible, and thus also a genetical interpretation of the glacier deposits in the lower Rhine area.

On the basis of the deposits of ground moraine material between strata of glacio-fluvial sands, three periods of ice advance could be proved to have taken place in the Louisendorf-Moyland region during the Drenthe.

\section{Einleitung: Forschungsstand und Problemstellung}

Im Saale-Glazial (Drenthe-Stadial) erreichte das Inlandeis das untere Niederrheingebiet und stauchte die untere Mittelterrasse zu Wällen auf. Eisvorstöße und Stauchwallbildungen sind von Tноме $(1958,1959)$ ausführlich beschrieben worden. Beim Vorstoß sowie beim Rückzug des Eises lagerten die Schmelzwässer glazifluviatile Kiese und Sande (Sander) ab. Stauchwälle und Sander bilden den Niederrheinischen Höhenzug. Dieser wurde beim Rückzug des Rheins in sein altes Bett größtenteils erodiert, so daß heute von den Stauchwällen und Sanderterrassen nur noch Fragmente vorzufinden sind. Die paläo-

*) Anschrift des Autors: Dipl.-Geogr. Dr. H. S i e b e r tz, Seminar für Geographie, Pädagogische Fakultät, Universität Bonn, Römerstr. 164, 5300 Bonn. 
geographische Entwicklung des unteren Niederrheingebietes ist unter Berücksichtigung der neuesten Erkenntnisse in der Quartärforschung von Heine \& SiEBERTz (1980) beschrieben worden.

Der Niederrheinische Höhenzug erstreckt sich von Krefeld bis Kleve-Kranenburg auf deutscher Seite. Die Stauchwälle um Kleve-Kranenburg und Kalkar (Moyland) mit der ausgeprägten Sanderterrassen im Raum Louisenburg-Uedem (Abb.1) sind morphologisch gut erhalten. In der Vergangenheit ist dieser Raum unter den verschiedensten quartärgeologischen und -morphologischen Aspekten untersucht worden (vgl. BRAUN 1956, 1964, 1968, 1978a, b; Heine 1983; Heine \& Siebertz 1980; Siebertz 1980, 1983).

Günstige Aufschlußverhältnisse gewährten in jüngster Zeit im Raum LouisendorfMoyland (Abb.4) einen guten Einblick in die quartärstratigraphischen Verhältnisse auf dem nördlichen Niederrheinischen Höhenzug. Die gewonnenen Erkenntnisse konnten im Rahnen der geomorphologischen Kartenaufnahme 1:25000 Blatt Kalkar ${ }^{1}$ ) sinnvoll

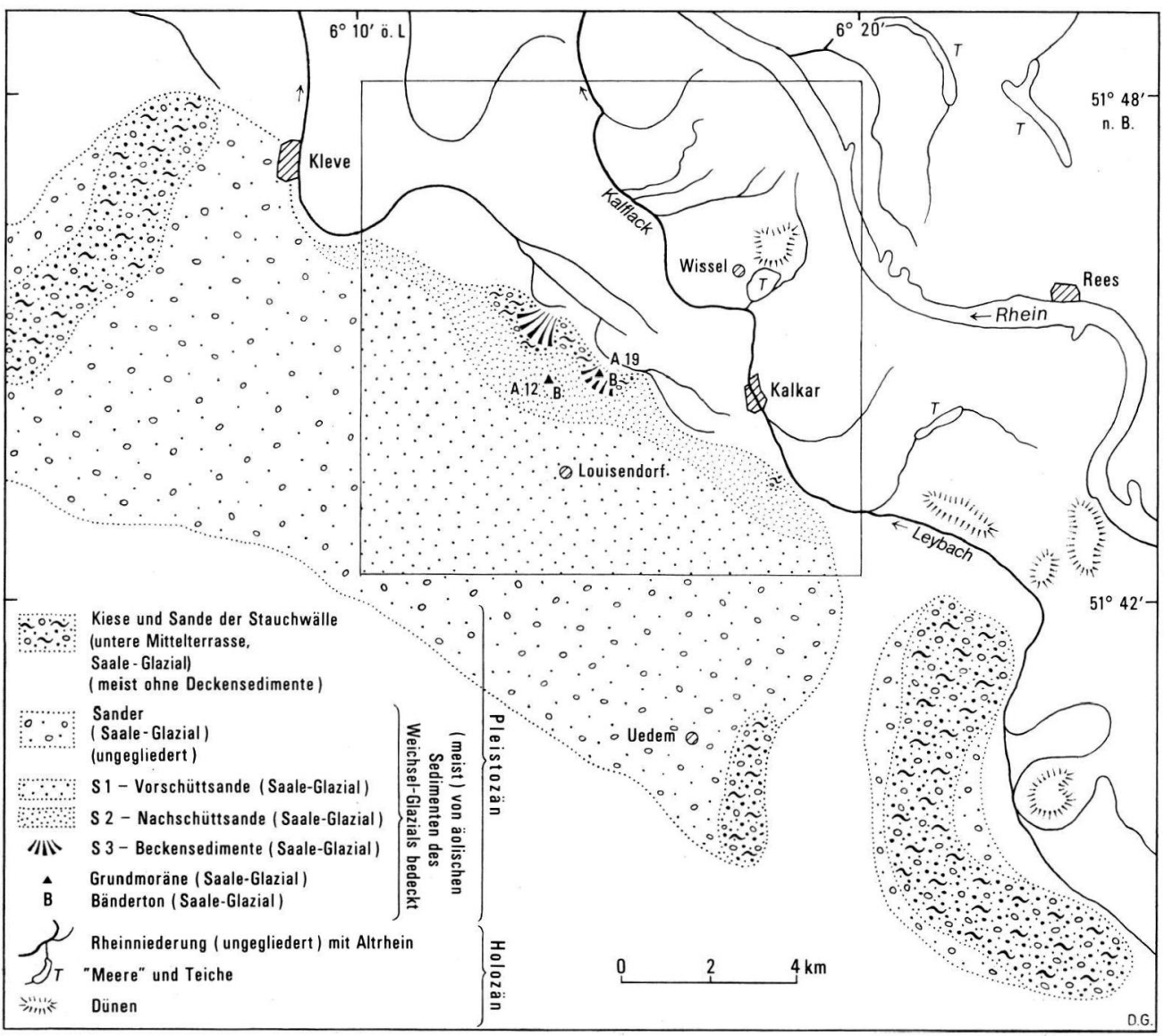

Abb. 1: Geologisch-geomorphologische Übersichtskarte des nördlichen Niederrheinischen Höhenzuges mit Kartenausschnitt Blatt Kalkar.

1) DFG-Forschungsprojekt Geomorphologische Detailkartierung in der Bundesrepublik Deutschland. 
verwandt werden, so daß sich für das Kartenblatt eine detaillierte Sandergliederung durchführen ließ (vgl. Siebertz 1980), welche die glazialmorphologischen Prozesse in ihrer Abhängigkeit von den Sedimentablagerungen widerspiegelt.

Die Sandersedimente lassen sich u. a. durch ihre stratigraphische Lage zur Grundmoräne gliedern (vgl. Heck 1961). Das geringe Vorkommen an Grundmoräne in den pleistozänen Sedimenten am unteren Niederrhein erschwert aber die Gliederung des Sanders. Mit Hilfe typischer Streubereiche in der Korngrößenzusammensetzung war es allerdings möglich, ein differenziertes Bild von der Sanderzusammensetzung zu gewinnen (Abb. 1).

Das Vorkommen lokaler Grundmoränenreste und deren Einbettung in die Sandersedimente im Aufschluß A 12 (Abb. 1, 5, 6; R 25.15350; H 57.34200) läßt die glazialmorphologische Entwicklung nicht nur vom stratigraphischen, sondern auch vom sedimentologischen Gesichtspunkt aus erläutern. Damit bietet sich für die Aussagefähigkeit quartärmorphologischer Prozesse nicht nur die Chronostratigraphie an, sondern mit Hilfe des

Sedinnents läßt sich 1. die räumliche Verbreitung gliedern und 2. die morphogenetische Entwicklung dieses Raumes darstellen.

\section{Methodische Utberlegungen zur Gliederung des Sanders}

Aufgrund von Untersuchungen im Aufschluß A 19 (R 25.16600; H 57.34300) bei Moyland (Abb. 1) hat Braun (1964) erkannt, daß die glazifluviatilen Schmelzwasserbildungen differenziert zusammengesetzt sind. BRAUN (1964, 1978a) gliedert ältere und jüngere Schmelzwassersande und -kiese, wobei er die jüngeren Ablagerungen als Beckensedimente bezeichnet ${ }^{2}$ ).

Die Profillinie von Braun (1964, 1978a) läßt erkennen, daß die Grenze zwischen älteren und jüngeren Sandersedimenten etwa mit der von Srebertz (1980) kartierten Grenze übereinstimmt, welche die unterschiedlich zusammengesetzten Sanderablagerungen voneinander trennt (Abb. 1; Beilage 1 in Siebertz 1980).

Eine Gesetzmäßigkeit in den glaziären Ablagerungen sieht HECK (1961). Er faßt sie in einem glaziären Zyklus zusammen. Beim Vorrücken sowie beim Rückzug des Eises werden durch die Schmelzwässer Kiese und Sande abgelagert. Der Zyklus beginnt im Liegenden mit (gröberen) Vorschüttsanden, darüber folgt (als Ablagerung des Hochglazials) eine mehr oder weniger block- und schuttbeladene Grundmoräne, auf der beim Abtauen des Gletschers (feinere) Nachschüttsande abgelagert werden. Dieser Zyklus wird im Hangenden bei der Toteisbildung von Beckensedimenten (Sande, Schluffe und Tone, meist Bändertone) abgeschlossen (HECK 1961).

Diese Sedimentabfolge nennt Heck (1961) eine Phase in der idealen glaziären Sequenz. Sie hat (vornehmlich) stratigraphischen Charakter. Demnach lassen sich Vor- und Nachschüttsande nur durch eine eingelagerte Grundmoräne gliedern. HEMPEL (1981) vertritt die Meinung, daß entsprechend Sedimentgehalt, Art der Ablagerung und äußerer

2) Braun (1964, 1978a) gliedert ältere (gestauchte) und jüngere (ungestauchte) Schmelzwasserablagerungen (vgl. Profil). Hierbei handelt es sich um die älteren gröberen Sedimente bei Louisendorf (Vorschüttsande nach Braun 1968) und die jüngeren feineren Sedimente (Nachschüttsande bzw. Beckensedimente nach Braun 1968) im Aufschluß bei Moyland (A 19). Diese jüngeren Schmelzwasserablagerungen in A 19 werden nach BRAUN (1968) von den unteren gestauchten sowie den oberen ungestauchten Schmelzwassersedimenten aufgebaut. Die Schwermineraluntersuchungen (Braun 1964, 1978a) beziehen sich nur auf gestauchte und ungestauchte Schmelzwasserablagerungen in A 19, und nicht auf den älteren nach Braun (1964, 1978a) gestauchten Sander (= Vorschüttsand) bei Louisendorf. 
Form Vorschüttsande, Grundmoräne, Nachschüttsande, Endmoränen, Oser und Kamesformen unterschieden werden können, was bei Sanderablagerungen ohne die eingelagerte Grundmoräne aber nicht immer möglich ist.

Der glaziäre Zyklus (Vorschüttsand, Grundmoräne, Nachschüttsand) als normale Ablagerungsfolge hat sich in der Praxis geologischen und geomorphologischen Arbeitens bewährt (vgl. Arnold 1977; Hempel 1980, 1981; Siebertz 1980; Thiermann 1970a, b). Sedimentologische Untersuchungen liegen über diesen Zyklus allerdings nicht vor, so daß beim Fehlen des stratigraphischen Aufbaus nur von (ungegliederten) Sandersedimenten gesprochen wird.

Selbst beim Fehlen der Grundmoräne sollten die Begriffe Vorschütt- und Nachschüttsand beibehalten werden, zumal sich mit Hilfe der unterschiedlichen Sedimentzusammensetzung die Vorstoß- und Rückzugsphasen des Eises (z. B. am unteren Niederrhein) klären lassen (vgl. Abb. 6).

Auf dem nördlichen Niederrheinischen Höhenzug hat SieberTz (1980) drei Sandersedimente unterschieden, deren Verbreitung in der morphologisch-morphochronologischen Karte von Kalkar und Umgebung dargestellt ist (Abb. 1; Beilage 1 in SieberTz 1980). Unterschieden wurden 1. (gröbere) Vorschüttsande $\left(\mathrm{S}_{1}\right)$, 2. (feinere) Nachschüttsande $\left(\mathrm{S}_{2}\right)$ und 3. feinsandig-schluffige Beckensedimente $\left(\mathrm{S}_{3}\right)$. Den Beckensedimenten sind lokal Bändertone untergeordnet, die in A 12 geringmächtig sind (Abb.6), in A 19 jedoch eine Mächtigkeit von mehreren Metern erreichen (SiEBERTz 1980). Charakteristische Kornsummenkurven sind mit den weichselzeitlichen Decksedimenten, die (meistens) den Sander bedecken, in Abb. 2 dargestellt (vgl. Sieber Tz 1983).

Mit Hilfe von Sedimentanalysen hat Siebertz (1980) versucht, für die einzelnen Sanderkomplexe charakteristische Korngrößenzusammensetzungen zu finden. So wurde für die Vorschüttsande $\left(S_{1}\right)$ ein Maximum von etwa $80 \%$ in der Mittelsandfraktion

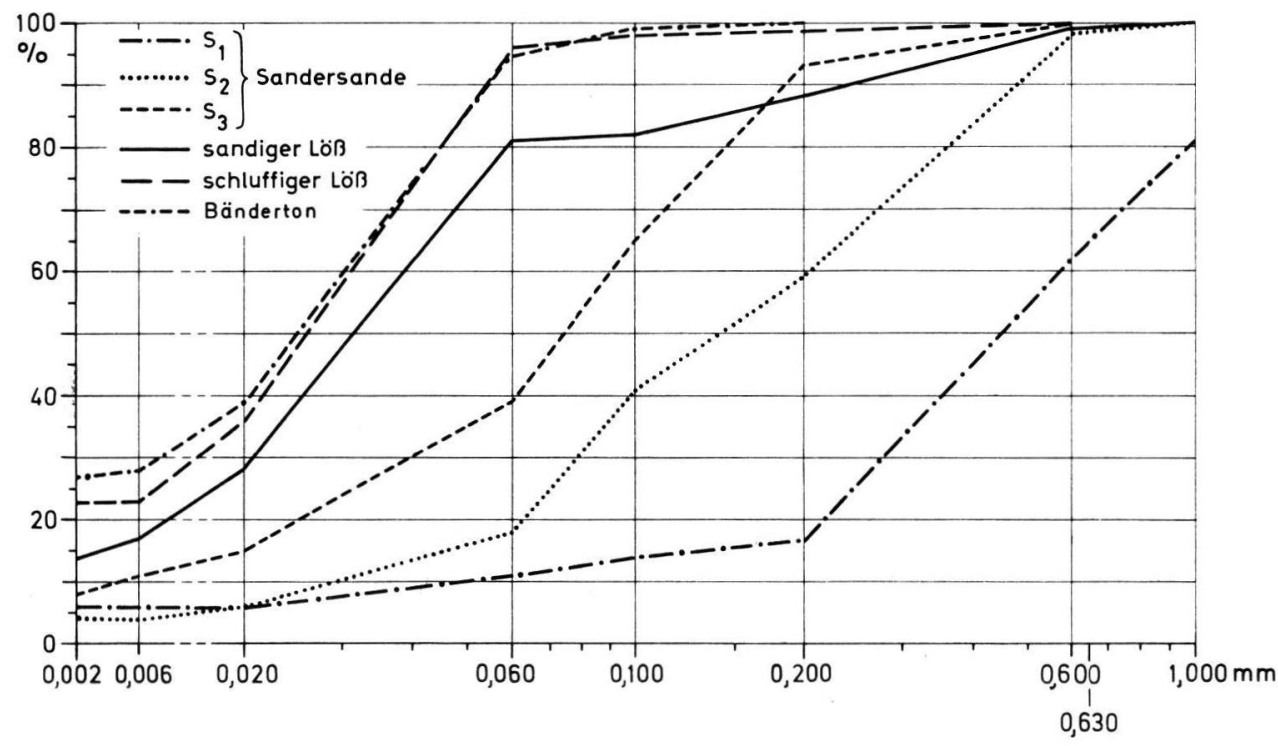

Abb 2: Kornsummenkurven der am Sanderaufbau beteiligten Sedimente und deren Deckschichten. 


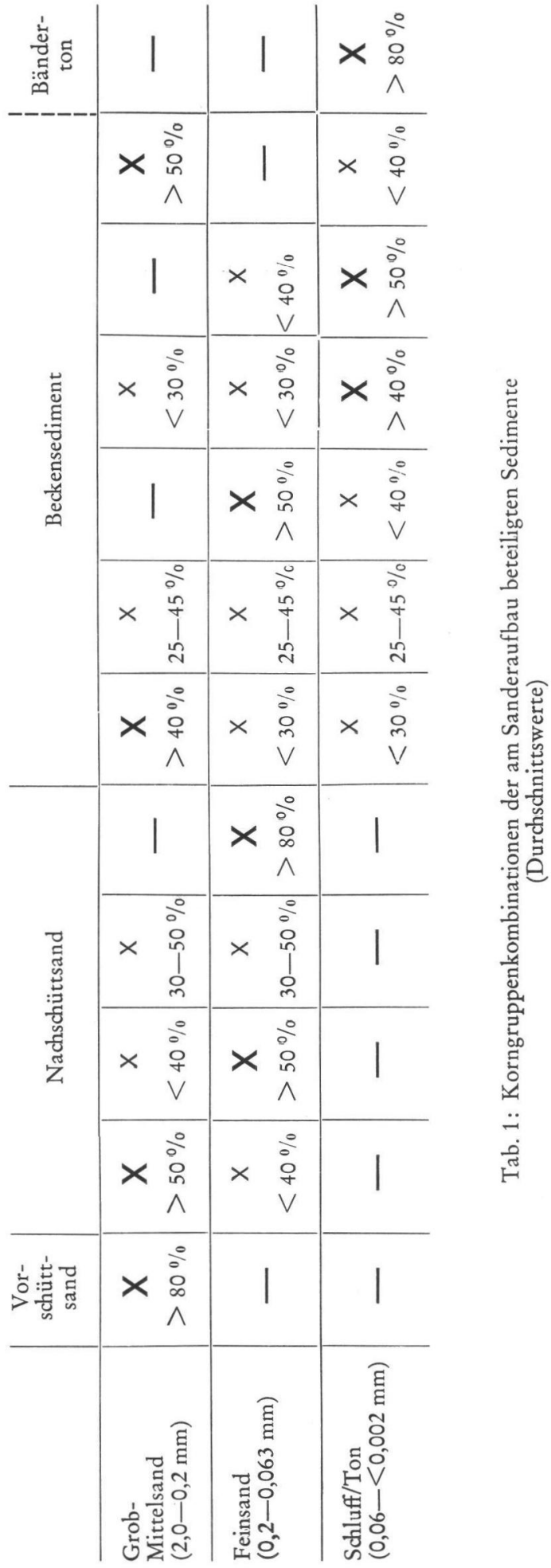


$(0,63-0,2 \mathrm{~mm})$, für die Nachschüttsande $\left(\mathrm{S}_{2}\right)$ etwa $60 \%$ im Mittelsand $(0,63-0,2 \mathrm{~mm})$ und etwa $30 \%$ im Feinsand $(0,2-0,063 \mathrm{~mm})$ ermittelt. Die Beckensedimente $\left(\mathrm{S}_{3}\right)$ werden von etwa $60 \%$ Feinsand $(0,2-0,063 \mathrm{~mm}$ ) und etwa 30\% Silt (Grobschluff 0,06$0,02 \mathrm{~mm}$ ) aufgebaut (SIEBERTZ 1980).

Neuere sedimentologische Untersuchungen lassen es ratsam erscheinen, eine erweiterte Klassifikation vorzunehmen. Es hat sich gezeigt, daß bei der Gegenüberstellung der Proben einzelne charakteristische Korngrößenfraktionen kaum eine Rolle spielen, sondern bestimmte Korngrößenfraktionen in Kombination auftreten und günstige Werte liefern, die eine Sandergliederung ermöglichen (vgl. Tab. 1).

Die Sandergliederung ist am günstigsten durchzuführen mit Hilfe der Kornfraktionenkombination, wie sie aus Tab. 1 ersichtlich ist. Es sind 1. die Siebfraktionen von Grobund Mittelsand $(2,0-0,2 \mathrm{~mm}), 2$. Feinsand $(0,2-0,063 \mathrm{~mm})$ sowie 3. die Schlämmfraktionen (Schluff und Ton 0,06- $<0,002 \mathrm{~mm})^{3}$ ).

Aus Tab. 1 ist zu ersehen, daß die Sandersedimente aus verschiedenen Korngruppen in unterschiedlichen Kombinationen zusammengesetzt sind. Dabei ergibt sich bei den Korngruppen ein höherer Prozentsatz (X) und (meistens) ein niedrigerer Prozentsatz (x), wobei (x) mit $\geqq 19 \%$ festgelegt ist. Daraus resultieren folgende Werte (Tab. 1):

1. glazifluviatile Vorschüt ts a nde mit einer klaren Dominanz im Grob-Mittelsand, wobei das Maximum deutlich im Mittelsand (0,63-0,2 $\mathrm{mm})$ liegt.

2. glazifluviatile $\mathrm{Nachs}$ ch ü t $\mathrm{ts}$ a $\mathrm{de}$ treten als Kombination Grob-Mittelsand mit Feinsand oder nur mit einem Maximum im Feinsand auf. Die Grob-MittelsandKombination ( $\mathbf{X})$ beinhaltet ihr Korngrößenmaximum im Mittelsand $(0,63-0,2 \mathrm{~mm})$, während der Feinsand ( $\mathbf{X})$ sein Maximum in der gröberen Feinsandfraktion (0,2$0,1 \mathrm{~mm}$ ) aufweist.

3. glazilimnische Beckensedimente beinhalten als neue Korngrößengruppe die Schluff-Tonfraktionen. Die Kombinationen sind aus Tab. 1 ersichtlich. Der GrobMittelsand ( $\mathbf{X})$ hat das Maximum im Mittelsand $(0,63-0,2 \mathrm{~mm})$; im Feinsand $(\mathbf{X})$ ist entweder ein ausgeglichenes Maximum zwischen den beiden Feinsandfraktionen $(0,2-0,1 \mathrm{~mm} ; 0,1-0,063 \mathrm{~mm})$ vorhanden oder ein deutliches Maximum beim feineren Feinsand $(0,1-0,063 \mathrm{~mm})$. Die Schluff-Ton-Kombination $(\mathbf{X})$ hat ihr Maximum im Grobschluff $(0,06-0,02 \mathrm{~mm})$.

4. glazilimnische $\mathrm{B}$ ä n d e r t o n e liegen mit einem deutlichen Korngrößenmaximum im Grobschluff $(0,06-0,02 \mathrm{~mm})$.

Die unterschiedlichen Korngrößengruppen (1-4 in Tab. 1), welche den Sander des Kartenblattes Kalkar aufbauen (Abb. 1; Beilage 1 in Siebertz 1980), sind aus dem Konzentrationsdreieck (Abb. 3) zu ersehen.

Nachschüttsande und Beckensedimente gehen im Gelände übergangslos ineinander über. Dies ist aus glazialmorphologischen Gründen auch verständlich; beide Sedimentkörper sind genetisch an die Abschmelzzeit des Eises gebunden. Selbst in den zahlreichen Bohrungen im Stauchwall bei Moyland (Abb.4) verzahnen sich Nachschüttsande und Beckensedimente, so daß die flächenhafte Ausdehnung der Beckensedimente nicht zu lokalisieren war. Lediglich dort, wo eine kumulative Häufung von Beckensedimenten nach-

$\left.{ }^{3}\right)$ Korngrößenfraktionen nach DIN 4188 (vgl. LESER 1977). 


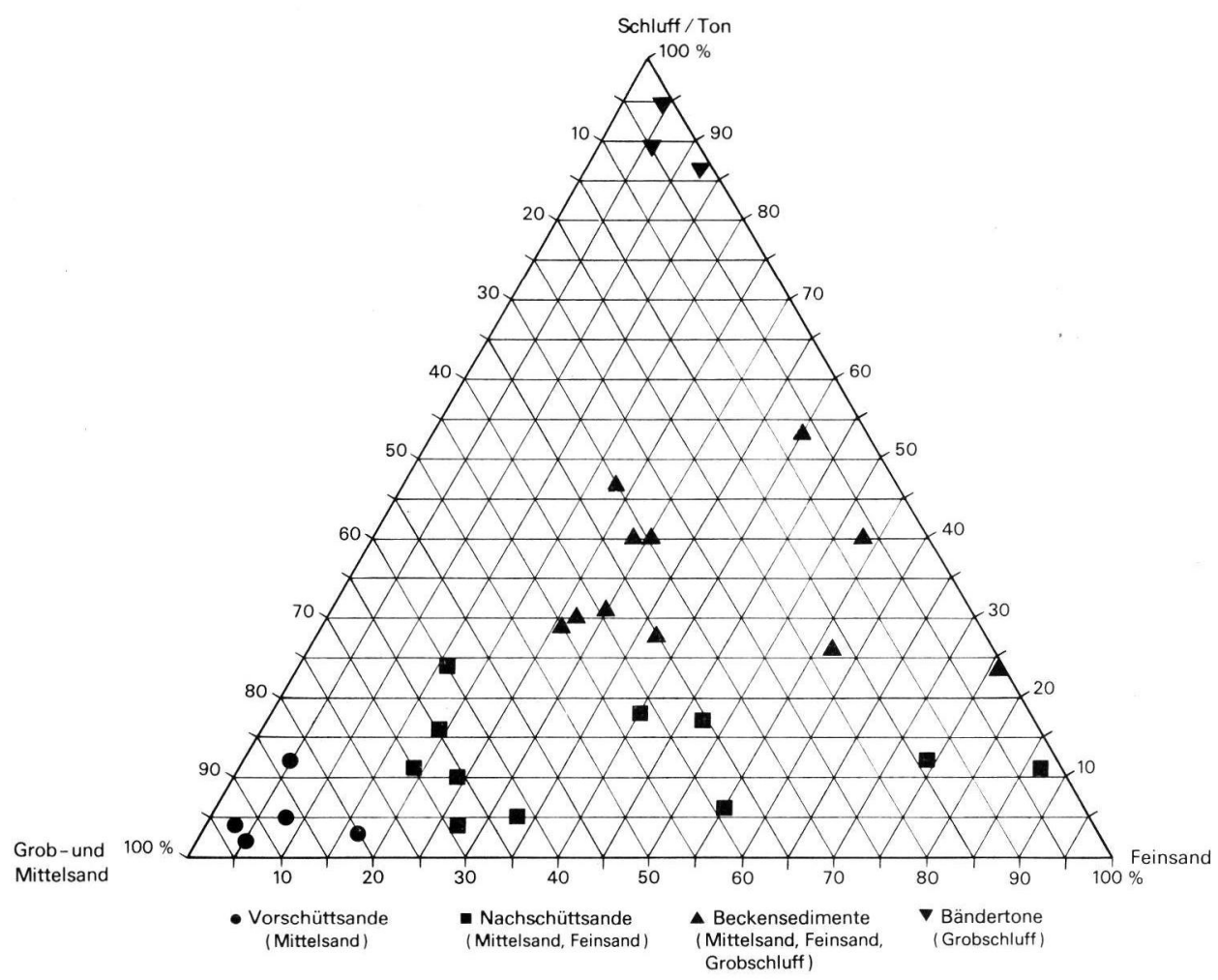

Abb. 3: Korngrößengruppen der am Sanderaufbau des Kartenblattes Kalkar beteiligten Sedimente.

zuweisen war, wurde eine Schwemmfächersedimentation abgebildet (Abb. 1; Beilage 1 in SiEBERTZ 1980) ${ }^{4}$ ).

\section{Die Bedeutung der Gliederung von Eisrandablagerungen für glazialmorphologische Prozesse}

Der glaziäre Zyklus hat für die Eisrandablagerungen am unteren Niederrhein Konsequenzen. Diese sind bedingt 1 . durch die maximale Eisausdehnung im Raum Louisendorf (Abb. 1; Beilage 1 in Siebertz 1980) und 2. durch die Sander- und Grundmoränenablagerungen. $\mathrm{Da}$ die Grundmoräne im allgemeinen wenig verbreitet ist (vgl. BRAUN 1959), wird die Gliederung des glaziären Zyklus erschwert.

$\left.{ }^{4}\right)$ Glazilimnische Sedimente (Beckensedimente oder Stauseeablagerungen) werden beim Eiszerfall abgelagert. Sie bestehen aus Sand, Schluff und Ton. Bei den Tonablagerungen handelt es sich meistens um gebänderte Sedimente (Bändertone) oder um Warven, wenn sie einen deutlichen jahreszeitlichen Rhythmus aufweisen.

Vom unteren Niederrhein sind nur geringe Vorkommen von Bändertonablagerungen bekannt. Dagegen haben die sandig-schluffigen Ablagerungen eine größere räumliche Verbreitung und stehen vom Substrat her den Sandersedimenten wesentlich näher als die Bändertone, die zu diesen von der Zusammensetzung her keine unmittelbare Beziehung aufweisen (vgl. Abb. 3).

Der Bänderton hat ferner geologisch-geomorphologisch eine größere Bedeutung im Rahmen der Geochronologie, so daß es ratsam ist, sein Vorkommen als eigenständiges Sediment neben den sandig-schluffigen Beckensedimenten aufzuführen. 


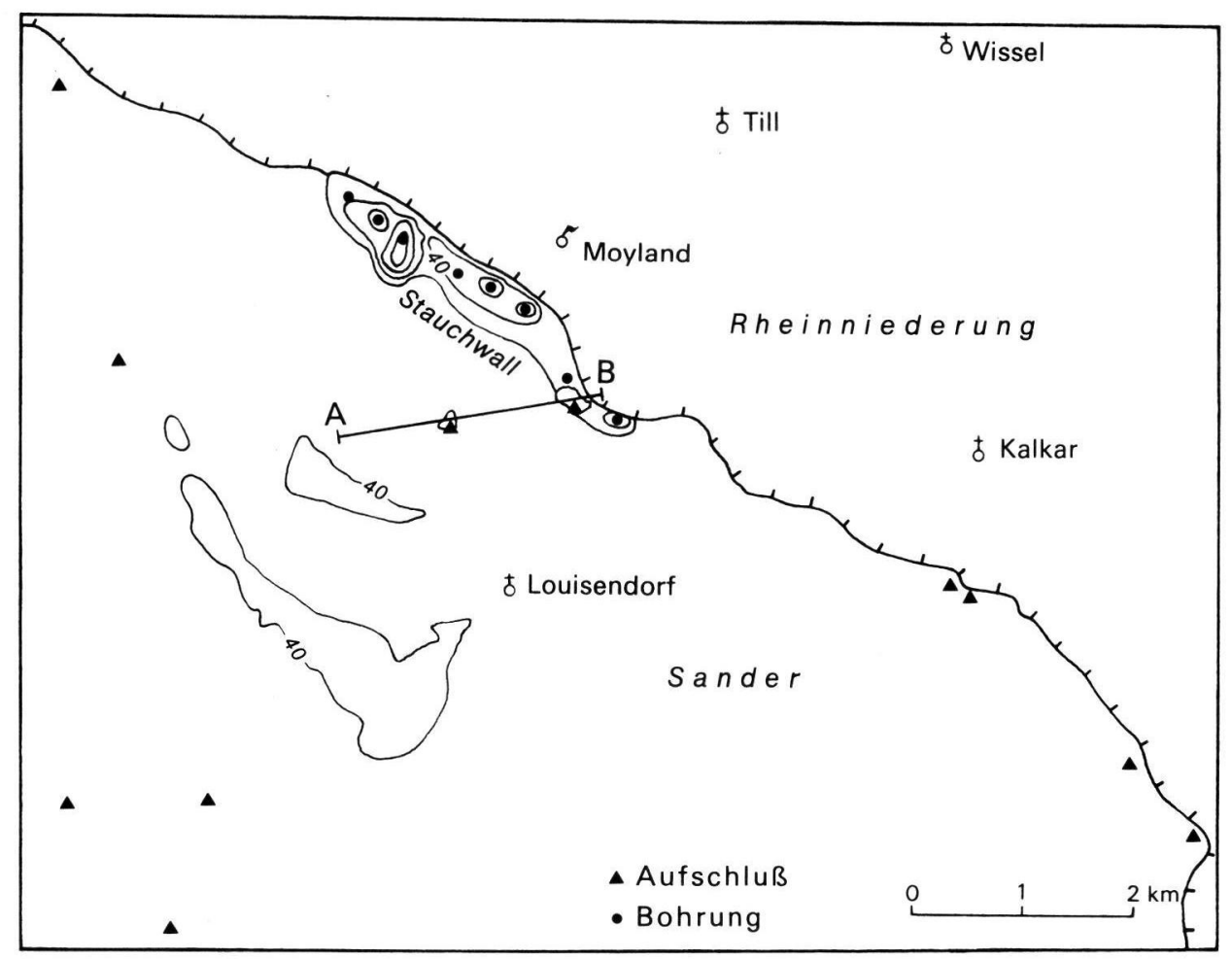

Abb. 4: Bohr- und Aufschlußkarte von Stauchwall und Sander des Kartenblattes Kalkar.

Untersuchungen von BRAUN (1964) haben ergeben, daß in A 19 bei Moyland Grundmoräne als Ausfüllung von Mulden und Rinnen auftritt. Im Rahmen der Kartierung des Blattes Kalkar von SieberTz (1980) konnte in A 19 gestauchte Grundmoräne über Beckensedimenten, durch Bohrungen im Stauchwall sowie als geschlossenes größeres Vorkommen in A 12 nachgewiesen werden (Abb. 1, 5, 6; Beilage 1 in Siebertz 1980). Damit ließ sich erstmals Grundmoräne in größerem Umfange in den Stauchwällen im Raum LouisendorfMoyland nachweisen. Die Mächtigkeit in der Aufschlußwand A 19 betrug etwa 1,001,50 m (Siebertz 1980), während in A 12 die Mächtigkeit nur wenige Dezimeter betrug (Abb. 6).

Die Einbettung der Grundmoräne in die unterschiedliche Sanderfazies macht die Deutung der genetischen Entwicklung im Raum Louisendorf kompliziert. Das Aufschlußprofil (A 12) in Abb. 6 zeigt die sedimentologische und stratigraphische Abfolge. Aufgrund dieser Erkenntnisse ergeben sich für die Genese folgende Befunde:

(1) Während des Drenthe-Eisvorstoßes (Amersfoorter-Stadium) erreichte das Inlandeis den unteren Niederrhein (vgl. Thомe 1958, 1959). Seine maximale Ausdehnung erreichte der Gletscher im Raum Kalkar bei Louisendorf (Louisendorf-Staffel) mit der Vorschüttsand-Ablagerung (Abb. 1,5). Die Grenze der maximalen Eisausdehnung des ersten Eisvorstoßes fällt bei Louisendorf geomorphologisch mit der Vorschüttsanderbildung zusammen. Das abfließende Schmelzwasser hat in diesem Raum isolierte Höhenrücken im 
A

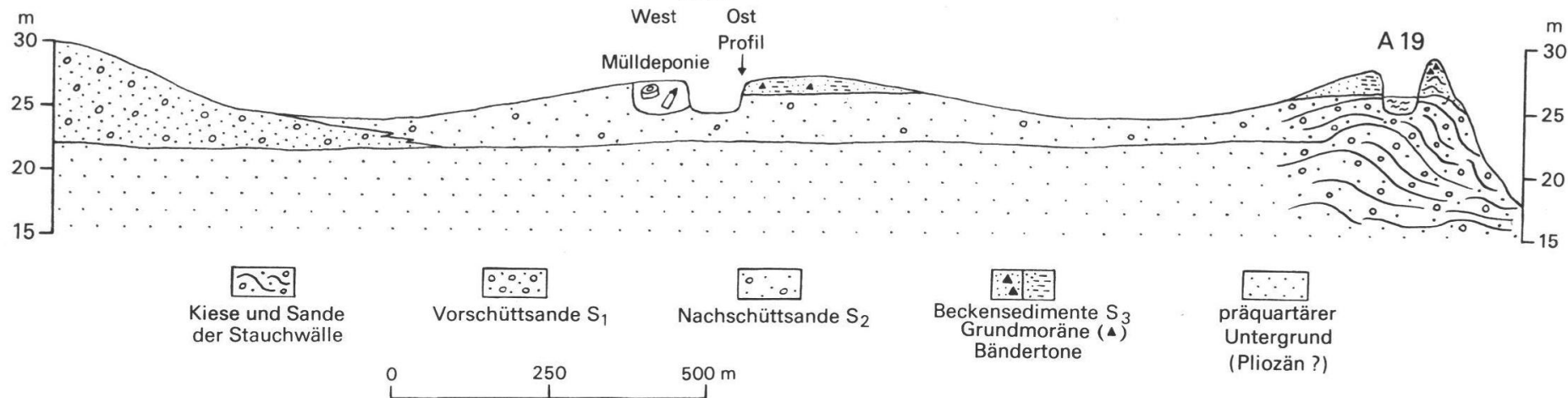

Abb. 5: Schematisches abgedecktes Querprofil durch die Sandersedimente des nördlichen Niederrheinischen Höhenzuges. 


\begin{tabular}{|c|c|c|c|c|}
\hline & & $\begin{array}{c}\text { Sedimentologische } \\
\text { Gliederung }\end{array}$ & $\begin{array}{l}\text { Stratigraphische } \\
\text { Gliederung }\end{array}$ & $\begin{array}{l}\text { Genetische } \\
\text { Gliederung }\end{array}$ \\
\hline \multirow[t]{2}{*}{$40 \mathrm{~cm}$} & $\overline{-}-\bar{\cdot}$ & sandiger Schluff (sU) & Sandlöß & äolische Ablagerungen \\
\hline & $\begin{array}{ll}-\div-1 \\
00 \\
\end{array}$ & Gerölle & Steinsohle & selektive Abtragung \\
\hline $\mathrm{Pg}-45 \mathrm{~cm}$ & $\begin{array}{l}0 \vdots \vdots \\
\vdots \vdots \vdots\end{array}$ & $\begin{array}{l}\text { Sand mit starken } \\
\text { Verbraunungen }(\mathrm{g} 1)\end{array}$ & Sander (Beckensediment) & $\begin{array}{l}\text { Eiszerfall (Toteisbildung mit Stausee- } \\
\text { ablagerungen) (als Fließerde überprägt?) }\end{array}$ \\
\hline $25 \mathrm{~cm}$ & $-0_{0}^{-1} \div \overline{4}^{-}$ & $\begin{array}{c}\text { tonig-sandig-kiesige Schicht } \\
\text { mit vereinzelt nordischen Gesteinen }\end{array}$ & Grundmoräne II & 3. Eisvorsto B (Moyland II-Staffel) \\
\hline P7 $-35 \mathrm{~cm}$ & 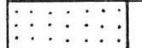 & grau-grüner Sand & Sander (Nachschüttsand) & Rückzug des Eises \\
\hline $15 \mathrm{~cm}$ & $\mid \Delta: \Delta: \vdots$ & $\begin{array}{l}\text { Sand mit Verbraunungen (g1) } \\
\text { und vereinzelt nordischen Gesteinen }\end{array}$ & Grundmoräne I & 2. Eisvorstoß (Moyland I-Staffel) \\
\hline $\mathrm{P}_{5}-12 \mathrm{~cm}$ & $\overline{-----=}$ & grau-brauner Ton & Bänderton & \multirow{5}{*}{$\begin{array}{r}\text { Eiszerfall (Toteisbildung) } \\
\text { (Stauseeablagerungen) }\end{array}$} \\
\hline $\mathrm{P}_{4}-28 \mathrm{~cm}$ & & grüner Sand & \multirow{2}{*}{ Sander (Beckensediment) } & \\
\hline $30 \mathrm{~cm}$ & & grüner Sand mit Verbraunungen (g1) & & \\
\hline $1,5 \mathrm{~cm}$ & 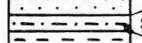 & sandig-toniger Verbraunungsabschnitt (g1) & & \\
\hline $\mathrm{P}_{2}-27 \mathrm{~cm}$ & 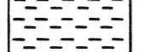 & Ton (pseudovergleyt g1, g2) & Bänderton & \\
\hline \multirow{2}{*}{$\mathrm{P}_{1}-\geqq 5 \mathrm{~m}$} & 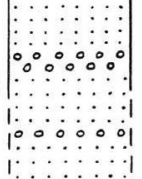 & \multirow[t]{2}{*}{$\begin{array}{l}\text { grau-blauer (glazifluviatil um- } \\
\text { gelagerter tertiärer ?) Sand } \\
\text { mit kleinen Kiesbändern durchsetzt }\end{array}$} & $\begin{array}{c}\text { Sander } \\
\text { (Nachschüttsand) }\end{array}$ & Rückzug des Eises \\
\hline & 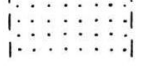 & & $\begin{array}{l}\text { Sander (Vorschüttsand } \\
\text { von Louisendorf) }\end{array}$ & $\begin{array}{c}\text { 1. Eisvorsto } \beta \\
\text { (Louisendorf - Staffel) }\end{array}$ \\
\hline
\end{tabular}

Aufnahme: April 1979

Abb. 6: Profil A 12 (R25.15350; H57.34200).

$40 \mathrm{~m}$ Niveau herausmodelliert (Abb. 4; Beilage 1 in Siebertz 1980). Braun (1968) sah in ihnen glazialtektonische Stauchrücken (vgl. geologische Karte), wofür keine Anhaltspunkte vorliegen. Die Morphologie (Höhenrücken und Anlage der Schmelzwasserrinnen) zeigt deutlich, daß es sich hierbei um Erosionsrücken handelt (SIEBERTz 1980).

Der Stauchwall bei Moyland hat seine heutige Form nicht beim ersten Eisvorstoß erhalten. Diese ist auf den Einfluß eines späteren Vorstoßes zurückzuführen. Die zahlreichen nordischen Gesteine, die aus der Tiefe in A 19 gefördert wurden (vgl. Braun 1964, 1978a), lassen den Schluß zu, daß ihre Ablagerung an die Louisendorf-Staffel gebunden war. Sie kann als Grundmoräne des ersten Eisvorstoßes gesehen werden, die vom Eis überfahren wurde, während der Gletscher vor Louisendorf zum Stehen kam und die Schmelzwässer die Sanderhochfläche aufbauten (Beilage 1 in SiEBERTz 1980).

(2) Mit dem Abschmelzen des Eises wurde der Nachschüttsand abgelagert (Abb. 1, 5). Er wurde in seiner Ausdehnung kartiert und reicht bis in den Stauchwall bei Moyland hinein (Abb. 1; Beilage 1 in Siebertz 1980). In A 12 konnte er an der Basis des Profils nachgewiesen werden (Abb. 6: P 1 mit 39\% Grob-/Mittelsand und 55\% Feinsand); in A 19 bildet er nach BRAUN (1968) den basalen (gestauchten) Schmelzwassersand.

Mit dem Eiszerfall bildeten sich Stauseeablagerungen, die als Beckensedimente (vornehmlich Feinsande, Schluffe und Tone) ausgebildet sind und auf den Nachschüttsanden liegen (Abb. 6: P 2 mit 95\% Schluff/Ton; P 4 mit 76\% Feinsand und 24\% Schluff/Ton; P 5 mit $13 \%$ Feinsand und $87 \%$ Schluff/Ton). In A 19 sind die Beckensedimente, die nach BraUN (1968) als jüngere (ungestauchte) Schmelzwassersande bezeichnet werden, 
sehr mächtig und besitzen eine relativ große räumliche Ausdehnung (Schwemmfächerbildung in Abb. 1 und Beilage 1 in Siebertz 1980). Die Bändertone erreichen in A 19 ihre größte Mächtigkeit von etwa $2 \mathrm{~m}$.

Beckensedimente sind im allgemeinen immer in abflußlosen Becken, die vermutlich von Toteis eingeschlossen waren, abgelagert. Ahnliche Ablagerungen von Feinsand und Bänderton, sedimentiert unter gleichen Bedingungen, wurden von CROMMELIN \& MAARLEVELD (1952) in der Veluwe beschrieben.

(3) Es erfolgte ein zweiter Eisvorstoß (Moyland I-Staffel), der nicht die Ausdehnung des ersten erreichte. Mit diesem Gletschervorstoß ist die Bildung des heutigen Stauchwalls bei Moyland verbunden (Abb. 1; Beilage 1 in Siebertz 1980). Die Beckensedimente in A 19 wurden dabei von einer recht mächtigen Grundmoräne bedeckt und lokal gestaucht ${ }^{5}$ ). Das Eis erreichte A 12 (Abb. 1) und lagerte die Grundmoräne I ab (Abb. 5, 6). Die Höhe von Louisendorf wurde von dem zweiten Gletschervorstoß nicht mehr erreicht, denn die Vorschüttsande weisen eine ungestörte Lagerung auf.

Mit dem Abschmelzen des Eises wurde ein geringmächtiger Nachschüttsand (Abb. 6: P 7 mit $87 \%$ Feinsand) abgelagert, der von einem dritten (schwachen) Eisvorstoß (Moyland II-Staffel) überfahren wurde und die Grundmoräne II ablagerte (Abb.6). Diese wurde im Hangenden bei der Toteisbildung von Beckensedimenten (Abb. 6: P 9 mit 44\% Grob-/Mittelsand, 26\% Feinsand, 30\% Schluff/Ton) abgeschlossen, womit sich schließlich der endgültige Eiszerfall einstellte. Die Schichten P 2 bis P7 (Abb.6) sind leicht gestaucht.

\section{Diskussion}

Braun (1978a), Heine (1983) und Thome (1959) sind der Annahme, daß zwei Eisvorstöße für das glazialmorphologische Bild am unteren Niederrhein verantwortlich sind, wovon dem ersten Eisvorstoß die größte Bedeutung zukommt. Unklar bleibt allerdings, wie weit die Einflüsse des ersten Eisvorstoßes für die glaziale Bildung der Stauchwälle und Sanderflächen im Raum Louisendorf-Moyland von Bedeutung sind.

\begin{tabular}{|c|c|c|c|c|c|}
\hline $\begin{array}{l}\text { Warthe- } \\
\text { Stadial }\end{array}$ & \multicolumn{2}{|c|}{$\begin{array}{c}\text { Krefelder } \\
\text { Mittelterrasse }\end{array}$} & \multirow{2}{*}{$\begin{array}{l}\text { Thome } \\
\text { (1959) }\end{array}$} & \multirow{2}{*}{$\begin{array}{l}\text { Kaiser \& } \\
\text { Schütrumpf } \\
\quad(1960)\end{array}$} & \multirow{2}{*}{$\begin{array}{c}\text { Siebertz } \\
(1983)\end{array}$} \\
\hline $\begin{array}{l}\text { Gerdau- } \\
\text { Interstadial }\end{array}$ & \multicolumn{2}{|c|}{ Erosion } & & & \\
\hline \multirow{2}{*}{$\begin{array}{l}\text { Drenthe- } \\
\text { Stadial }\end{array}$} & \multirow{2}{*}{$\begin{array}{l}\text { untere } \\
\text { Mittel- } \\
\text { terrasse }\end{array}$} & \multirow{2}{*}{ 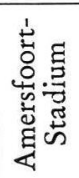 } & $\begin{array}{c}\text { Kamper- } \\
\text { Staffel }\end{array}$ & $\begin{array}{l}\text { Mintarder- } \\
\text { Staffel }\end{array}$ & $\begin{array}{l}\text { Moyland-II } \\
\text { Moyland-I } \\
\text { Staffel }\end{array}$ \\
\hline & & & $\begin{array}{l}\text { Neußer- } \\
\text { Staffel }\end{array}$ & $\begin{array}{l}\text { Krefelder- } \\
\text { Staffel }\end{array}$ & $\begin{array}{l}\text { Louisendorf- } \\
\text { Staffel }\end{array}$ \\
\hline
\end{tabular}

Tab. 2: Eisvorstöße am unteren Niederrhein im Saale-Glazial

$\left.{ }^{5}\right)$ Die Stauseeablagerungen in A 19 weisen lokal Stauchungen und Fältelungen auf (Abb. 5 in BraUn 1964), obwohl sie von BraUn (1968) als jüngere (ungestauchte) Sandersedimente bezeichnet wurden. Entnommene Proben zeigen, daß es sich um Beckensedimente handelt.

9 Eiszeitalter u. Gegenwart 
Der erste Eisvorstoß reichte mit seiner maximalen Eisausdehnung bis Louisendorf. Dieser Vorstoß ist mit der Neußer-Staffel von Thоме (1959) und der Krefelder-Staffel von Kaiser \& Schütrumpf (1960) zu vergleichen (Tab. 2). Heine (1983) nimmt an, daß mit dem ersten Eisvorstoß der Stauchwall bei Moyland gebildet wurde, und ein zweiter, allerdings schwacher Eisvorstoß zum Überfließen des Stauchwalls bei Moyland führte, vor der Höhe von Louisendorf zum Stehen kam und dessen Schmelzwässer den älteren Sander aufbauten.

Die Bildung des Stauchwalls durch den ersten Eisvorstoß ist jedoch zweifelhaft, weil der beiLouisendorf einsetzende ältereSander (Vorschüttsande) mit einer durchschnittlichen Mächtigkeit von über $5 \mathrm{~m}$ (im Raum Louisendorf bis etwa $10 \mathrm{~m}$, nach $W$ leicht abnehmend und erreicht in den Schmelzwasserrinnen bei Pfalzdorf oberhalb der Niersniederung etwa $2-3 \mathrm{~m}$ ) die gesamte Sanderhochfläche zwischen Kleve-LouisendorfUedem aufbaut; die Vorschüttsande haben von allen am Aufbau beteiligten Sedimenten den größten Anteil und die größte flächenhafte Ausdehnung. Der in Abb. 1 dargestellte ungegliederte Sander besteht fast nur aus Vorschüttsanden; Nachschüttsande haben selbst im Raum der Kleve-Kranenburger Stauchwälle - eine geringe lokale Verbreitung und spielen dort am Sanderaufbau eine untergeordnete Rolle. Die größte flächenhafte Verbreitung haben die Nachschüttsande im Kartenblatt Kalkar (Abb.1; Beilage 1 in SiEBERTZ 1980).

Die Ablagerung dieser Vorschüttsande (Mächtigkeit, flächenhafte Ausdehnung) ist nicht durch Schmelzwässer des zweiten (schwachen) Eisvorstoßes erfolgt. Dies wird auch belegt durch die recht mächtige und räumlich ausgedehnte Nachschüttsand-Ablagerung des zurückweichenden Eises in Abb. 1. Ferner sprechen die morphologisch kräftig ausgebildeten Schmelzwasserrinnen, die westlich von Louisendorf in die Niersniederung hin entwässerten sowie die im Halbkreis um das $40 \mathrm{~m}$ Höhenniveau von Louisendorf angelegten, zur Rheinniederung hin verlaufenden Rinnen, für Entwässerungslinien, die auf einen kräftigen Eisvorstoß hindeuten (Beilage 1 in SIEBERTz 1980). Auch konnten östlich von Louisendorf keine Vorschüttsande mehr nachgewiesen werden.

Im Raum Louisendorf fehlt zwar die Grundmoräne zum ersten Eisvorstoß. Dafür schließt der glaziäre Zyklus mit den Nachschüttsanden (P 1 in Abb.6) und den darüber liegenden Stauseeablagerungen (P 2, P 4, P 5 in Abb.6).

Der Annahme von Braun (1964, 1978a), daß der ältere Sander um Louisendorf gestaucht sei, wurde bereits von SieberTz (1980) widersprochen. Die Höhenrücken wurden als Erosionsformen der abfließenden Schmelzwässer gedeutet (vgl. Beilage 1 in SieberTz 1980); die in Abb. 7 (Seite 60 in Braun 1968) dargestellte Stauchung, welche die Veranlassung zu einem dem heutigen Stauchwall im Westen bei Moyland vorgelagerten Stauchrücken gab, ist nicht belegt. Der Vorschüttsand bei Louisendorf liegt ungestört, was von HEINE (1983) bestätigt wird.

Ein zweiter Eisvorstoß im Raum Moyland wird auch von HeINe (1983) angenommen. Er bemerkt allerdings, daß es sich hierbei um eine kleine Besonderheit am Eisrand der Neußer-Staffel von Thоме (1959) handelt. Dieser Eisvorstoß wird nach Heine (1983) durch die Grundmoräne dokumentiert; sie ist in A 12 (Abb. 5,6) zwar geringmächtig, wird im glaziären Zyklus aber durch eigenständige Sandersedimente, wenn auch geringmächtige Nachschüttsande, belegt (Abb. 6).

Der zweite Eisvorstoß (Moyland I-Staffel) kann durchaus als eigenständiger, wenn auch schwächerer Vorstoß gewertet werden, der nicht die Ausdehnung des ersten erreichte. Er ist mit der Kamper-Staffel (Thome 1959) bzw. Mintarder-Staffel (KaISER-SchüтRUMPF 1960) zu vergleichen (Tab. 2), wenn ihm (vielleicht) auch nicht die morphologische Bedeutung der Kamper-Staffel von Tноме (1959) zukommt. 
Mit diesem zweiten Eisvorstoß ist die eigentliche Bildung des heutigen Stauchwalls bei Moyland verbunden. Dabei wurden die Nachschüttsande (unterer gestauchter jüngerer Schmelzwassersand nach BRAUN 1968 in A 10) sowie die Stauseeablagerungen (Beckensedimente), Ablagerungen der Louisendorf-Staffel in A 19 (Abb.1), lokal gestaucht und stellenweise von einer Grundmoräne überzogen, die in A 12 (Abb.5,6) noch als geringmächtige Grundmoräne I zu finden ist.

Der hohe Anteil nordischer Geschiebe (Grundmoräne der Louisendorf-Staffel) in A 19 (BRAUN 1964, 1968, 1978a), der während der Kiesbaggerei aus der Tiefe gefördert wurde, ist bereits beim ersten Eisvorstoß abgelagert, vom Eis überfahren und von Schmelzwasserablagerungen zugeschüttet worden, so daß diese Geschiebe derzeit in keiner Aufschlußwand anstehen.

Für die Niederlande (Veluwe und Geldersche Vallei) sind von Edelman \& MaARleVELD (1958) drei Eisvorstöße unterschieden worden, die dem Amersfoorter-Stadium angehören. Ein dritter (lokaler) Eisvorstoß (Moyland II-Staffel) kann durch die Grundmoräne II (Abb. 6) belegt werden. Bei diesem Vorstoß kann es sich wahrscheinlich nur um einen schwachen Eisvorstoß handeln, welcher für die Glazialtektonik vermutlich unbedeutend war.

GRIPP (1951) geht von Einzeluntersuchungen der letzten Jahre aus, die belegen, daß die Stauchwälle selten auf einmal, häufig in mehreren Anläufen gestaucht wurden. Welchem Eisvorstoß die Stauchung der den Beckensedimenten auflagernden Grundmoräne in A 19 zuzuschreiben ist, bleibt zunächst ungeklärt. Die Annahme von Braun (1978a), daß nach der Bildung der Stauchwälle und nach Ablagerung der älteren Sedimente (Vorschüttsande bei Louisendorf) ein erneuter Eisvorstoß zur Stauchung dieser älteren Sedimente führte, läßt sich nicht belegen (vgl. Heine 1983).

\section{Schlußwort}

Das Ergebnis zeigt, daß sedimentologische Untersuchungen eine horizontale sowie vertikale Gliederung von Eisrandablagerungen ermöglichen. Mit ihnen ist eine Deutung der morphogenetischen Prozesse möglich, die sonst nur durch die stratigraphischen Verhältnisse erkannt werden oder mit Hilfe der Glazialtektonik interpretierbar erscheinen.

Aufgrund granulometrischer Analysen konnten in den Eisrandablagerungen am unteren Niederrhein Vorschütt-, Nachschüttsande und Stauseeablagerungen (Sande, Schluffe und Bändertone) unterschieden werden, die aufgrund ihrer Lagerungsverhältnisse und der eingelagerten Grundmoräne im Raum Louisendorf-Moyland drei Eisvorstöße aufzeigen. Vorschüttsand, Grundmoräne und Nachschüttsand gehören einem glaziären Zyklus an; diese sind für den Eisvorstoß und den Eisrückzug charakteristisch. Drei Staffeln (Louisendorf, Moyland I und II) lassen sich aufgrund der morphologischen und sedimentologischen Gegebenheiten im Raum des Kartenblattes Kalkar nachweisen. 


\section{Schriftenverzeichnis}

Annold, H. (1977): Geologische Karte Nordrhein-Westfalen $1: 100000$, Erläuterungen zu Blatt C 4314 Gütersloh, 156 S., 31 Abb., 10 Tab., 1 Taf.; Krefeld (Geol. L.-Amt Nordrhein-Westfalen).

Braun, F. J. (1956): Die Terrassengliederung am linken Niederrhein zwischen Geldern und Kalkar. - Geologie en Mijnbow (N. S.), 18: 374-378, 3 Abb.; s'Gravenhage.

- (1959): Endmoränen, Terrassen und holozäne Ablagerungen bei Xanten am Niederrhein. Fortschr. Geol. Rhld. u. Westf., 4: 247-254, 2 Abb., 1 Tab., 1 Taf.; Krefeld.

- (1964): Endmoränen-Stauchwall und Eisrandbildungen bei Moyland/Ndrh. - Der Niederrhein, 2: 58-63, 7 Abb., 1 Tab.; Krefeld.

- (1968): Übersichtskarte von Nordrhein-Westfalen $1: 100000$, Erläuterungen zur geologischen Karte C 4302 Bocholt, 13-92, 12 Abb., 4 Tab., 4 Taf.; Krefeld (Geol. L.-Amt NordrheinWestfalen).

- (1978a): Geschiebekundliche und mineralogisch-petrologische Besonderheiten im EndmoränenStauchwall von Moyland bei Kalkar/Ndrh. - Fortschr. Geol. Rhld. u. Westf., 28: 325333, 2 Abb., 1 Tab., 1 Taf.; Krefeld.

- (1978b): Zur Herkunft und Zusammensetzung des "Sandlösses“ auf der Uedemer SanderHochfläche (Niederrhein). - Fortschr. Geol. Rhld. u. Westf., 28: 335—343, 5 Abb., 3 Tab.; Krefeld.

Crommelin, R. D. \& Maarleveld, G. C. (1952): Fluvioglazialer Bänderton der nördlichen Veluwe (Niederlande). - Abhandl. Naturw. Verein Bremen, 33: 133-142, 2 Fig.; Bremen.

Edelman, C. H. \& MaArleveld, G. C. (1958): Pleistozän-geologische Ergebnisse der Bodenkartierung in den Niederlanden. - Geol. Jb., 73: 639-684, 26 Abb., 3 Tab., 2 Taf.; Hannover.

GripP, K. (1951): Über den morphologischen Nachweis großer Schwankungen des Eisrandes. Eiszeitalter u. Gegenwart, 1: 65-69, 2 Abb.; Óhringen.

Heck, H. L. (1961): Glaziale und glaziäre Zyklen. - Geologie, 10: 378-395, 2 Abb.; Berlin.

HeINE, K. (1983): Der Vorstoß des nordeuropäischen Inlandeises am Niederrhein (Raum KleveKalkar-Goch). - Beiträge zum Quartär der nördlichen Rheinlande, Arb. z. Rhein. Landeskde, 51: 39-49, 4 Abb.; Bonn.

- \& Siebertz, H. (1980): Abriß der paläogeographischen Entwicklung des unteren Niederrheingebietes. - Niederrheinische Studien, Arb. z. Rhein. Landeskde, 46: 1-13, 6 Abb., 1 Tab.; Bonn.

Hempel, L. (1980): Der „Osning-Halt“ des Drenthe-Stadials am Teutoburger Wald im Lichte neuerer Beobachtungen. - Eiszeitalter u. Gegenwart, 30: 45-62, 4 Abb., 5 Tab.; Hannover.

- (1981): Erläuterungen zur geomorphologischen Karte 1:25000 der Bundesrepublik Deutschland, GMK 25 Blatt 6, 3814 Bad Iburg, 53 S., 8 Abb., 11 Tab., 1 Kt.; Berlin.

KAISER, K. H. \& SchütrumpF, R. (1960): Zur Gliederung mittel- und jungpleistozäner Schichten in der Niederrheinischen Bucht. - Eiszeitalter u. Gegenwart, 11: 166-185, 5 Abb., 2 Tab.; OOhringen.

Leser, H. (1977): Feld- und Labormethoden der Geomorphologie. - 446 S., 91 Abb., 28 Tab.; Berlin (de Gruyter).

Siebentz, H. (1980): Ausgewählte quartärmorphologische Probleme am unteren Niederrhein. Ergebnisse einer geomorphologischen Kartierung, dargestellt am Beispiel einer geomorphologischen Ubersichtskarte vom Raum Kalkar. - Niederrheinische Studien, Arb. z. Rhein. Landeskde, 46: 37-46, 3 Abb., $2 \mathrm{Kt}$; Bonn.

- (1983): Neue sedimentologische Untersuchungsergebnisse von weichselzeitlichen äolischen Decksedimenten auf dem Niederrheinischen Höhenzug. - Beiträge zum Quartär der nördlichen Rheinlande, Arb. z. Rhein. Landeskde, 51: 51-97, 8 Abb., 6 Tab., 1 Kt.; Bonn.

Thiermann, A. (1970a): Geologische Karte Nordrhein-Westfalen 1:25000, Erläuterungen zu Blatt 3712 Tecklenburg, 243 S., 22 Abb., 10 Tab., 7 Taf.; Krefeld (Geol. L.-Amt Nordrhein-Westfalen).

- (1970b): Geologische Karte Nordrhein-Westfalen 1:25000, Erläuterungen zu Blatt 3711 Bevergern, 120 S., 9. Abb., 6 Tab., 5 Taf.; Krefeld (Geol. L.-Amt Nordrhein-Westfalen).

Thоме, K. N. (1958): Die Begegnung des nordischen Inlandeises mit dem Rhein. - Geol. Jb., 76: 261-308, 11 Abb.; Hannover.

- (1959): Eisvorstoß und Flußregime an Niederrhein und Zuider See im Jungpleistozän. Fortschr. Geol. Rhld. u. Westf., 4: 197-246, 19 Abb., 5 Tab., 1 Taf.; Krefeld. 